\title{
Mitochondrial ROS contribute to oridonin-induced HepG2 apoptosis through PARP activation
}

\author{
XIAONING LIU, JINGJING KANG, HUI WANG and TAO HUANG \\ Department of Biochemistry, School of Medicine, Huanghe College of Science and Technology, \\ Zhengzhou, Henan 450063, P.R. China
}

Received November 9, 2016; Accepted November 10, 2017

DOI: $10.3892 / \mathrm{ol} .2017 .7665$

\begin{abstract}
Oridonin, the main active constituent of Rabdosia rubescens, is known to exert antitumor activity via the induction of apoptosis in numerous types of human cancer cells. However, the underlying regulatory mechanisms of mitochondrial ROS in oridonin-induced HepG2 apoptosis remain largely unknown, due to limitations of subcellular imaging resolution. Previously, it has been suggested that mitochondria serve a potential role in sensing and signaling cellular redox changes in vital biological processes such as cell death and the abiotic stress response, based on studies involving the mitochondrial-targeted redox-sensitive green fluorescent protein (GFP). To address this, a mitochondrial-targeted Grx1-roGFP2 (mtGrx1-roGFP2) biosensor was implemented to monitor real-time mitochondrial redox changes of HepG2 cells in response to either $\mathrm{H}_{2} \mathrm{O}_{2} /$ DTT or oridonin/SS31 treatment. It was determined that oridonin caused a perturbation in mitochondrial redox status, which in turn contributed to oridonin-induced apoptosis. Furthermore, a novel mechanism underlying the regulation of mitochondrial redox changes in oridonin-induced HepG2 apoptosis, presumably dependent on PARP cleavage, was proposed. In conclusion, the present study provides evidence in support of mitochondrial redox changes as a potential mediator in the apoptotic activities of oridonin in HepG2 cells, which provides insight into the molecular mechanisms by which mitochondrial redox signaling regulates oridonin-induced apoptosis in cancer therapy, and the development of mitochondria-specific oridonin as a promising novel anticancer therapeutic strategy.
\end{abstract}

Correspondence to: Dr Xiaoning Liu, Department of Biochemistry, School of Medicine, Huanghe College of Science and Technology, 666 Zijingshan South Road, Zhengzhou, Henan 450063, P.R. China

E-mail: xiaoningliu2016@126.com

Key words: oridonin, apoptosis, mitochondrial reactive oxygen species, mitochondrial redox changes, HepG2, mitochondrial-targeted glutaredoxin1-redox-sensitive green fluorescent protein 2, poly adenosine 5'-diphosphate ribose polymerase

\section{Introduction}

Apoptosis, or programmed cell death, is an ordered and orchestrated cellular process that occurs in physiological and pathological conditions $(1,2)$. Evasion of apoptosis serves a vital function in carcinogenesis and numerous novel treatment strategies that may restore the apoptotic signaling pathways towards homeostasis may be feasible for eliminating cancer cells $(3,4)$. Reactive oxygen species (ROS), as signals and modulators for cellular redox changes, are key mediators in the regulation of cell apoptosis $(5,6)$. Modulation of the redox state may potentially trigger apoptotic signaling pathways by cellular FLICE-like inhibitory protein degradation, apoptosis signal-regulating kinase 1 activation, cytochrome c release and interaction of caspase 9/Apoptotic protease-activating factor 1 (7). Previous studies (8-10) concerning the extrinsic and intrinsic pathways of apoptosis have focused on mitochondria, which are the major source of ROS generation and serve a central function in apoptosis regulation (11). It has been suggested that instead of elevating overall cellular ROS, regulation of mitochondrial ROS during apoptosis may be a promising therapeutic strategy in the treatment of various types of cancer (7).

As the most abundant cellular redox buffer, glutathione (GSH) is distributed in at least 9 different subcellular compartments, and protects cells against ROS through the oxidation of GSH to glutathione disulfide (GSSG). The reduced to oxidized glutathione ratio [GSH/GSSG] may be measured to reflect cellular redox changes (12-14). Alternatively, various redox-sensitive fluorescent dyes, such as dihydrodichlorofluorescein $\left(\mathrm{H}_{2} \mathrm{DCF}\right)$ have been frequently used to monitor ROS levels in a number of studies focusing on natural drug-induced apoptosis in cancer $(15,16)$. However, conventional approaches lack either spatiotemporal resolution or specificity resolution (17). This is a critical knowledge gap that has adversely limited the ability to comprehensively probe into the underlying regulatory mechanisms of mitochondrial ROS in natural drug-induced apoptosis in cancer, and has also impeded the development of novel redox-based therapeutic strategies targeting cancer. Previously, genetically-encoded redox-sensitive green fluorescent protein (roGFP) probes have been developed with a suitable range of redox potentials to measure the spatial and temporal intracellular oxidation status by ratiometric analysis, which is compartmental, 
real-time, reversible and measures dynamic variation trends compared with other methods for assessing intracellular redox status (13,18-20). Previously, human glutaredoxin1 (Grx1) was fused to the roGFP2 biosensor to facilitate specific real-time equilibration between the sensor protein and the glutathione redox couple, consequently allowing dynamic live imaging of the intracellular glutathione redox potential in different cellular compartments, with unprecedented sensitivity and temporal resolution $(14,21)$. Therefore, the ratiometric Grx1-roGFP2 biosensor targeted to mitochondria may be extremely useful in quantifying mitochondrial redox changes associated with natural drug-induced apoptosis in cancer.

Oridonin, a natural and safe ent-kaurene diterpenoid compound isolated from the plant Rabdosia rubescens, has been demonstrated to induce ROS to mediate apoptosis in multiple types of cancer, including human laryngeal cancer cells (22), human cervical carcinoma HeLa cells (23), hepatocellular carcinoma cells $(24,25)$, human epidermoid carcinoma A431 cells (26,27), human histiocytic lymphoma U937 cells (28), esophageal cancer KYSE-150 cells (29) and rheumatoid arthritis fibroblast-like synoviocytes (30). Furthermore, the combined use of AG1478 and oridonin augments the production of ROS and apoptosis $(22,26)$; Therefore, targeting epidermal growth factor receptor combined with oridonin may be a potentially effective anti-neoplastic therapy. Notably, neither study $(31,32)$ demonstrated that oridonin-induced apoptosis may be conferred by compartmental redox changes in live cells. In the present study, using live-cell imaging, it was demonstrated that the mitochondrial-targeted glutaredoxin1-redox-sensitive green fluorescent protein 2 (mtGrx1-roGFP2) probe expressed in HepG2 cells may be used as a real-time reporter of dynamic redox changes caused by oridonin. The possible regulatory mechanisms of mitochondrial redox signaling contributing to oridonin-induced apoptosis in HepG2 cells were also assessed.

\section{Materials and methods}

Reagents and antibodies. Unless stated otherwise, reagents were purchased from Thermo Fisher Scientific, Inc. (Waltham, MA, USA). Oridonin $\left(\mathrm{C}_{20} \mathrm{H}_{28} \mathrm{O}_{6}, \mathrm{MW}=364.43\right.$; Chemical Abstracts Service registry number, 28957-04-2; lot, H1616026) was purchased from Shanghai Aladdin Bio-Chem Technology Co., Ltd. (Shanghai, China). The oridonin was dissolved in $\geq 99.7 \%$ dimethyl sulphoxide (DMSO; Sigma-Aldrich; Merck KGaA, Darmstadt, Germany) with $150 \mathrm{mM}$ as original stock solution. Subsequent to mixing by vortex $(1,500 \mathrm{rpm}$ for $10 \mathrm{sec}$ ), the solution was then filtered through a $0.22-\mu \mathrm{m}$ filter, which was then stored at $4^{\circ} \mathrm{C}$ prior to use. The solution was diluted in serum-free Dulbecco's modified Eagle's medium (DMEM; Gibco; Thermo Fisher Scientific, Inc.) medium prior to use. The maximum final concentration of DMSO in medium was maintained below $0.1 \%(\mathrm{v} / \mathrm{v})$ and did not exert any detectable effect on cell growth or cell death $(23,33)$. SS31, a small aromatic-cationic mitochondria-targeted peptide (H-D-Arg-Dmt-Lys-Phe- $\mathrm{NH}_{2}$ ), may be selectively concentrated in the inner mitochondrial membrane and reduce mitochondrial ROS generation (34-36). It was provided by Stealth BioTherapeutics, Inc., (Newton, MA, USA).

Primary antibodies against $\beta$-actin (catalog no. 8457P) and poly adenosine 5'-diphosphate ribose polymerase (PARP; catalog no. 9532T) were purchased from Cell Signaling Technology, Inc. (Danvers, MA, USA). A goat anti-rabbit immunoglobulin $\mathrm{G}$ horseradish peroxidase (HRP)-conjugated secondary antibody (catalog no. HS101-01) used for western blotting were purchased from Beijing Transgen Biotech Co., Ltd. (Beijing, China).

Gene constructs. The mtGrx1-roGFP2-containing vector (200 ng/ $\mu \mathrm{l}$ ) (21) was obtained from Professor Dick (German Cancer Research Center, Heidelberg, Germany). The mtGrx1-roGFP2 open reading frame was polymerase chain reaction Deutsches Krebsforschungszentrum, Im Neuenheimer Feld-amplified with the following primers: mt-forward, 5'-CGAGCTCAAGCTTCGAATTCGCCACCA TGGCCTCCACTCGTGTCCTC-3'; and mt-reverse, 5'-ACC CGGTAGAATTATCTAGATTACTTGTACAGCTCGTCC ATGC-3' by polymerase chain reaction (PCR), which was performed in a $20 \mu \mathrm{l}$ total reaction volume using the vector as DNA template and LongAmp ${ }^{\circledR}$ TaqDNA polymerase (New England BioLabs, Inc., Ipswich, MA, USA). The PCR reaction was run as follows: $94^{\circ} \mathrm{C}$ for $3 \mathrm{~min}$ as an initial denaturation step; amplification for 35 cycles with denaturation at $94^{\circ} \mathrm{C}$ for $30 \mathrm{sec}$, annealing at $58^{\circ} \mathrm{C}$ for $45 \mathrm{sec}$, and extension at $65^{\circ} \mathrm{C}$ for $90 \mathrm{sec}$; and final extension at $65^{\circ} \mathrm{C}$ for $10 \mathrm{~min}$. The mtGrx1-roGFP2 open reading frame was cloned into the pLenti-CMV-PGK-puro vector (Invitrogen; Thermo Fisher Scientific, Inc.) using EcoR I and $X$ ba I (20,000 U/ml; New England BioLabs, Inc.) to generate pLenti-CMV-mtGrx1-ro GFP2-PGK-puro vectors at $16^{\circ} \mathrm{C}$ for $12 \mathrm{~h}$. Restriction digestion was conducted with EcoR I and Xba I (20,000 units/ml; New England BioLabs, Inc.) at $37^{\circ} \mathrm{C}$ for $3 \mathrm{~h}$, and then DNA sequencing was performed by Openlab Co., Ltd. (Beijing, China) to verify the construction of a recombinant vector. The vector, along with the helper plasmids [pLP/VSVg, pLP1 and pLP2, (Invitrogen; Thermo Fisher Scientific, Inc.)], were used to prepare virus stocks for transduction experiments.

Stable cell line generation. The human hepatoblastoma HepG2 cell line $(37,38)$, obtained from the American Type Culture Collection (Manassas, VA, USA), was cultured in DMEM containing $10 \%$ (v/v) fetal bovine serum (Gibco; Thermo Fisher Scientific, Inc.) and antibiotics $(100 \mathrm{U} / \mathrm{ml}$ penicillin and $100 \mu \mathrm{g} / \mathrm{ml}$ streptomycin) at $37^{\circ} \mathrm{C}$ in an atmosphere containing $5 \% \mathrm{CO}_{2}$. HepG2 cells stably expressing the mtGrx1-roGFP2 biosensor were generated by lentiviral transduction (Thermo Fisher Scientific, Inc.) of the plasmids into interim 293T cells (American Type Culture Collection) for $12-24 \mathrm{~h}$ at $37^{\circ} \mathrm{C}$ and subsequent selection with $2 \mu \mathrm{g} / \mathrm{ml}$ puromycin (39). Colonies were screened for mtGrx1-roGFP2 expression by western blotting and Olympus IX71 reverse fluorescence microscopy (magnification, x100; Olympus Corporation, Tokyo, Japan) at an excitation wavelength of $488 \mathrm{~nm}$ with an emission filter of $507 \mathrm{~nm}$. For $12 \mathrm{~h}$ prior to treatment, the culture medium was altered to DMEM medium with $2 \%$ FBS to decrease the serum effect.

Cell viability assay. The viability of cells was measured with TransDetect ${ }^{\mathrm{TM}}$ Cell Counting Kit (CCK, catalog no. FC101-01; Beijing Transgen Biotech Co., Ltd., Beijing, China) according to the manufacturer's protocol (Beijing Transgen 
Biotech Co., Ltd.) with minor modifications. Briefly, $100 \mu 1$ HepG2-mtGrx1-roGFP2 cell suspension was dispensed into 96 -well plate $\left(5 \times 10^{3}\right.$ cells/well $)$ and the plate was pre-incubated at $37^{\circ} \mathrm{C}$ for $24 \mathrm{~h}$, followed by treatment with various concentrations of oridonin $(0,5,10,20,30$ or $60 \mu \mathrm{M})$ for $8 \mathrm{~h}$. Next, $10 \mu \mathrm{l}$ (100 $\mu \mathrm{l} / \mathrm{ml})$ of CCK working solution was added to each well of the plate and then incubated at $37^{\circ} \mathrm{C}$ for $2 \mathrm{~h}$. The absorbance of each well at $450 \mathrm{~nm}$ was measured under a multimode plate reader (PerkinElmer, Inc., Waltham, MA, USA). The results representing the average of 5 parallel samples were expressed as the relative percentage of cell growth inhibition.

Fluorescence microscopy. MtGrx1-roGFP2-expressing HepG2 cells placed in a Nest-35 confocal dish, where the temperature was maintained at $37^{\circ} \mathrm{C}$ by a stage top incubator (Tokai Hit Elmer, Inc., Waltham, MA, USA), were treated with $\mathrm{H}_{2} \mathrm{O}_{2}(1 \mathrm{mM})$ or dithiotheritol (DTT; $2 \mathrm{mM}$ ) and/or oridonin $(25 \mu \mathrm{M})$ or SS31 $(100 \mathrm{nM})$ and imaged using a Nikon A1R confocal laser scanning system (Nikon Corporation, Tokyo, Japan) equipped with a 0.95 numerical aperture (water immersion) objective lens at magnification $\mathrm{x} 40$. For excitation of 405-nm (channel 1) and 488-nm (channel 2), fluorescence was measured with a bandpass filter of 500-554 nm. Images were captured and exported to ImageJ software (version 1.50i; National Institutes of Health, Bethesda, MD, USA). Subsequent to setting thresholds to avoid low signal ratio artifacts, false-color ratio images were obtained by dividing the $405-\mathrm{nm}$ image by the 488-nm image on a pixel per pixel basis, as previously described (21).

To confirm the mitochondrial localization of the mtGrx1-roGFP2 probe in stable transfectants, the HepG2 cells expressing mtGrx1-roGFP2 were incubated with a pre-warmed $\left(37^{\circ} \mathrm{C}\right)$ rosamine-based MitoTracker probe $(100 \mathrm{nM}$; Thermo Fisher Scientific, Inc.) for $20 \mathrm{~min}$ at $37^{\circ} \mathrm{C}$ in an atmosphere of $5 \% \mathrm{CO}_{2}$ according to the manufacturer's protocol (Thermo Fisher Scientific, Inc.). Cells were visualized using Olympus FV1000 confocal laser scanning microscopy equipped with a magnification, $\mathrm{x} 40$ objective (Olympus Corporation) at $579 \mathrm{~nm}$ excitation and $599 \mathrm{~nm}$ emission wavelengths.

Analysis of apoptotic cells by flow cytometry. The percentage of apoptotic of cells exposed to oridonin $(25 \mu \mathrm{M})$ with or without SS31 (100 $\mathrm{nM})$ for $8 \mathrm{~h}$ were determined using a commercially available Annexin V-phycoerythrin (PE) apoptosis detection kit according to the manufacturer's protocol (BD Biosciences, Franklin Lakes, NJ, USA) with minor modifications. Briefly, cells were collected and washed twice in ice cold PBS and then resuspended in $100 \mu 11 \mathrm{X}$ binding buffer at a density of $1 \times 10^{5}$ cells $/ \mathrm{ml}$, and then incubated with $5 \mu \mathrm{l}$ Annexin V-PE and $5 \mu 1$ 7-aminoactinomycin (7-AAD) in the dark for $15 \mathrm{~min}$ at room temperature. Finally, $400 \mu 1$ of $1 \mathrm{X}$ binding buffer was added to each tube. Samples were analyzed using a BD FACSCalibur (BD Biosciences) and BD CellQuest Pro software (version 5.1; BD Biosciences) and evaluated based on the percentage of apoptotic cells that were Annexin V-PE-positive and 7-AAD-positive.

Observation of morphological changes. Apoptotic nuclear morphology was assessed using Hoechst 33342 (Beijing SolarbioScience \& Technology Co.,Ltd.,Beijing,China) in PBS buffer, as described previously (33). HepG2-mtGrx1-roGFP2 cells were grown in culture at $37^{\circ} \mathrm{C}$ for $12 \mathrm{~h}$. Following $12 \mathrm{~h}$ of incubation at $37^{\circ} \mathrm{C}$, the cells were perfused with oridonin $(25 \mu \mathrm{M})$ in the presence or absence of SS31 $(100 \mathrm{nM})$ at $37^{\circ} \mathrm{C}$ for $8 \mathrm{~h}$. For morphological observation, cells were incubated with Hoechst $33342(1 \mathrm{mg} / \mathrm{ml})$ for $25 \mathrm{~min}$ at $37^{\circ} \mathrm{C}$, washed and then examined using an Olympus FV1000 confocal laser scanning microscopy (Olympus Corporation) equipped with a x40 objective at $350 \mathrm{~nm}$ excitation and $461 \mathrm{~nm}$ emission wavelengths.

Western blot analysis. MtGrx1-roGFP2-expressing HepG2 cells were treated with oridonin $(25 \mu \mathrm{M})$ with or without SS31 $(100 \mathrm{nM})$ at $37^{\circ} \mathrm{C}$. After $8 \mathrm{~h}$, cells were washed twice with ice-cold PBS and solubilized in lysis buffer containing $20 \mathrm{mM}$ Tris (pH 7.5), $135 \mathrm{mM} \mathrm{NaCl}, 2$ mM EDTA, 2 mM DTT, $25 \mathrm{mM} \beta$-glycerophosphate, $2 \mathrm{mM}$ sodium pyrophosphate, $10 \%$ glycerol, $1 \%$ Triton $\mathrm{X}-100,1 \mathrm{mM}$ sodium orthovanadate (Sigma-Aldrich; Merck KGaA), $10 \mathrm{mM} \mathrm{Na}$ fluoride (NaF; Sigma-Aldrich; Merck KGaA), $10 \mu \mathrm{g} / \mathrm{ml}$ aprotinin, $10 \mu \mathrm{g} / \mathrm{ml}$ leupeptin and $1 \mathrm{mM}$ phenylmethane sulfonyl (PMSF; Sigma-Aldrich; Merck KGaA, ) for $30 \mathrm{~min}$ on ice. Lysates were centrifuged $(15,000 \mathrm{x} \mathrm{g})$ at $4^{\circ} \mathrm{C}$ for $15 \mathrm{~min}$. Soluble protein were denatured in SDS in a boiling water bath for 5 min, and quantified using Easy II Protein Quantitative Kit (BCA; catalog no. DQ111-01; Beijing Transgen Biotech Co., Ltd). A total of $10 \mu \mathrm{l}$ protein was electrophoresed on an 8-12\% gel using SDS-PAGE, and transferred to a nitrocellulose membrane. The membrane was blocked with $5 \%$ skim milk for $1 \mathrm{~h}$ at room temperature, then incubated overnight with the indicated primary antibodies against PARP (1:1,000 dilution; catalog no. $9532 \mathrm{~T}$ ) at $4^{\circ} \mathrm{C}$, prior to incubation with goat anti-rabbit IgG HRP-conjugated secondary antibody (1:10,000 dilution; catalog no. HS101-01) for $2 \mathrm{~h}$ at room temperature. Detection was performed using an EasySee Western Blot Kit (catalog no. DW101-01; Beijing Transgen Biotech Co., Ltd.).

Statistical analysis. All experimental data obtained from cultured cells are expressed as the mean \pm standard deviation. Statistical analysis was performed using SPSS (version 19.0; IBM Corp., Armonk, NY, USA). The data were analyzed using one-way analysis of variance, followed by Bonferroni's post hoc test. $\mathrm{P}<0.05$ was considered to indicate a statistically significant difference.

\section{Results}

Mitochondrial-targeted Grx1-roGFP2 biosensor functions in HepG2 cells. Previously, it was demonstrated that ROS induced by oridonin is capable of restoring apoptotic signaling pathways in HepG2 cells (32); however, whether mitochondrial ROS contribute to oridonin-induced apoptosis remains unknown. To monitor mitochondrial ROS in HepG2 cells during oridonin-induced apoptosis, stably transfected HepG2 cells were created with a Grx1-roGFP2 biosensor targeted to mitochondria. As demonstrated in Fig. 1A, the expressed Grx1-roGFP2 co-localized with the mitochondrial-selective probe following confocal laser scanning microscopy, indicating that Grx1-roGFP2 was correctly targeted to mitochondria in the transgenic cells. 
A
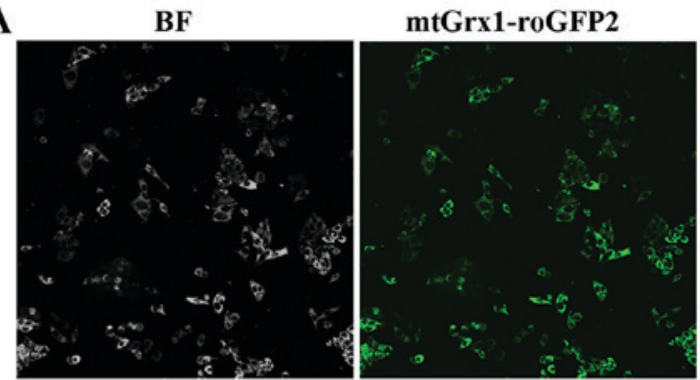

B

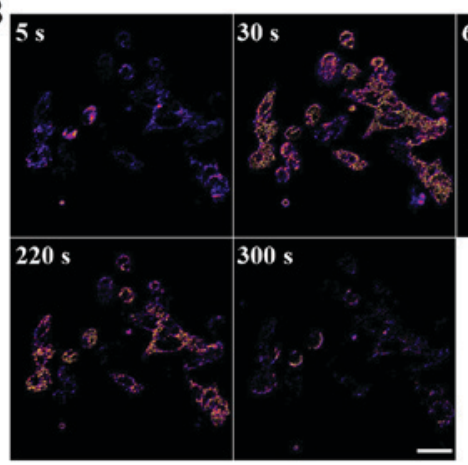

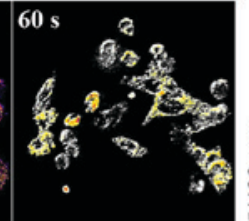

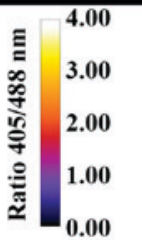

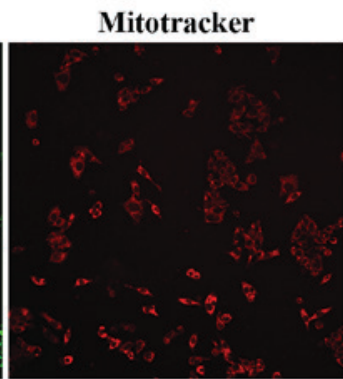
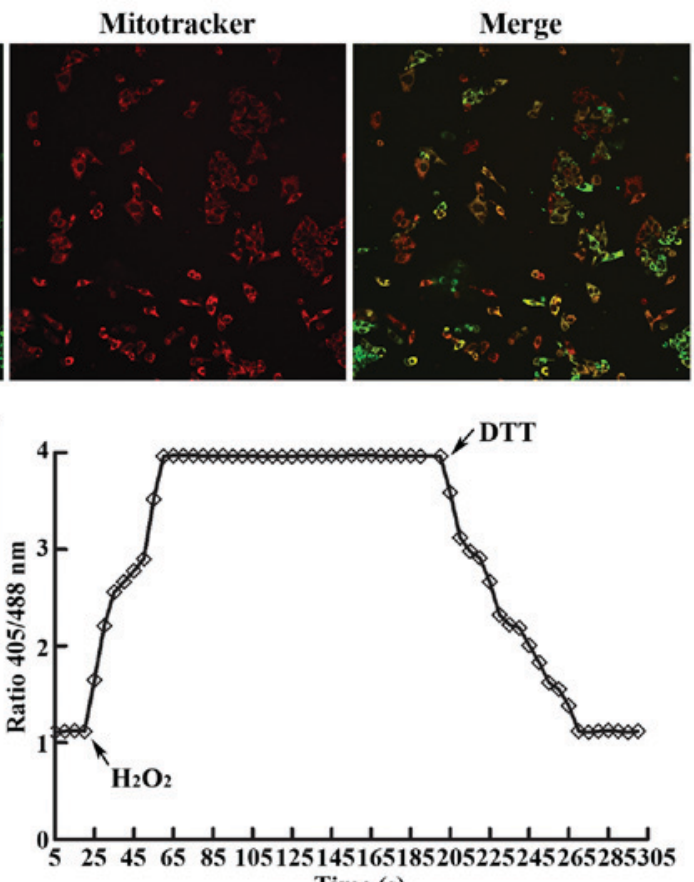

Time (s)

Figure 1. Generation of transgenic cell lines expressing the mtGrx1-roGFP2 biosensor. (A) Mitochondrial localization of Grx1-roGFP2 was performed by incubation with rosamine-based MitoTracker probe $(100 \mathrm{nM})$ for $20 \mathrm{~min}$, and then observation by Olympus FV1000 confocal laser scanning microscopy (magnification, x400). Fluorescence emission (488 nm for Grx1-roGFP2 and 599 nm for MitoTracker) micrographs demonstrated that the Grx1-roGFP2 sensor was targeted to mitochondria. (B) The mtGrx1-roGFP2 fusion protein allowed live imaging of rapid mitochondrial redox changes caused by $\mathrm{H}_{2} \mathrm{O}_{2}$ and DTT in HepG2 cells. HepG2 cells expressing mtGrx1-roGFP2 were imaged with excitation at $405 \mathrm{~nm}$ and $488 \mathrm{~nm}$ lasers respectively by Nikon A1R confocal laser scanning system (magnification, x400). After $20 \mathrm{sec}$, cells were perfused with $1 \mathrm{mM} \mathrm{H}_{2} \mathrm{O}_{2}$, followed by injection of 2 mM DTT for 180 sec. False-color ratiometric images created by dividing the 405-nm image by the 488-nm image at the indicated time points (left) are presented. Corresponding ratiometric data were plotted against time (right). Scale bar, $10 \mu \mathrm{m}$. Data are representative of 3 experiments. s, sec; DTT, dithiotheritol.

To determine whether Grx1-roGFP2 was responsive to mitochondrial redox changes and was able to be ratiometrically measured, HepG2 cells were treated with $1 \mathrm{mM} \mathrm{H}_{2} \mathrm{O}_{2}$, followed $180 \mathrm{sec}$ later by exposure to $2 \mathrm{mM}$ DTT. As indicated in Fig. $1 \mathrm{~B}$, the addition of $\mathrm{H}_{2} \mathrm{O}_{2}$ to the growth medium led to an immediate and marked response, with the 405/488 nm fluorescence ratios of Grx1-roGFP2 in the mitochondria of HepG2 cells ranging from 1.21 to 3.86 , which was maintained for $135 \mathrm{sec}$ until injection of DTT induced its reduction to 1.23. Taken together, these results revealed that Grx1-roGFP2 responded, as reflected in the altered $405 / 488 \mathrm{~nm}$ excitation fluorescence ratio, to mitochondrial redox changes triggered by $\mathrm{H}_{2} \mathrm{O}_{2}$ and DTT as authoritative oxidants and reductants, therefore providing a real-time detection system for dynamic and reversible changes to mitochondrial redox status induced by anticancer drugs.

Oridonin induces mitochondrial redox changes in HepG2 cells in a time-dependent manner. The primary aim of the present study was to establish a roGFP-based redox-sensing system to monitor redox changes in mitochondria, and to delineate the molecular mechanisms by which mitochondrial ROS control oridonin-induced apoptosis in HepG2 cells. To address this, Grx1-roGFP2 molecules targeting mitochondria, as aforementioned, were used to measure the perturbation of ROS levels reflected by the $405 / 488 \mathrm{~nm}$ excitation fluorescence ratio, as distinguished from the fluorescent intensity of ROS-specific probe $\mathrm{H}_{2} \mathrm{DCF}$, which has traditionally been used as the most applicable tool. However, $\mathrm{H}_{2} \mathrm{DCF}$ exhibits additional disadvantages, such as being destructive, integrated, irreversible, point-in-time and providing only a static redox status $(17,21,40)$. Representative fluorescence microscope images of mitochondrial Grx1-roGFP2 with corresponding ratiometric analysis are presented in Fig. 2. In the time course experiments, stimulation of HepG2 cells with oridonin at a concentration of $25 \mu \mathrm{M}$, responsible for a $\sim 50 \%$ inhibitory ratio at $8 \mathrm{~h}$ (Fig. 3), resulted in a 3 -fold increase in progressive oxidation of the sensor, which plateaued after $160 \mathrm{~min}$. As anticipated, SS31 prevented the oridonin-induced oxidation of mtGrx1-roGFP2, with a decline from 3.65 to 1.22 in the $405 / 488 \mathrm{~nm}$ excitation fluorescence ratio. In general, using mitochondria-localized Grx1-roGFP2, the present study has provided evidence that oridonin causes a disturbance of mitochondrial redox poise in a manner that is reversible and may be captured in real time.

Mitochondrial ROS mediate oridonin-induced apoptosis in HepG2 cells. To additionally explore whether mitochondrial ROS act as mediators in oridonin-induced apoptosis, HepG2 cells were co-incubated with SS31 (100 nM) and oridonin $(25 \mu \mathrm{M})$ for $8 \mathrm{~h}$ at $37^{\circ} \mathrm{C}$. Compared with untreated cells, a marked increase by almost $34.41 \%$ in cell apoptosis, as indicated by flow cytometry using an Annexin V-PE Apoptosis Detection kit (Fig. 4A), was observed in response to oridonin treatment. Furthermore, inhibition of apoptosis was induced by SS31, accompanied by a decrease in cell growth inhibition ratio from 38.87 to $5.75 \%$. Consistent with the apoptotic percentage, apoptotic morphology was detected by Hoechst 33342 staining. 


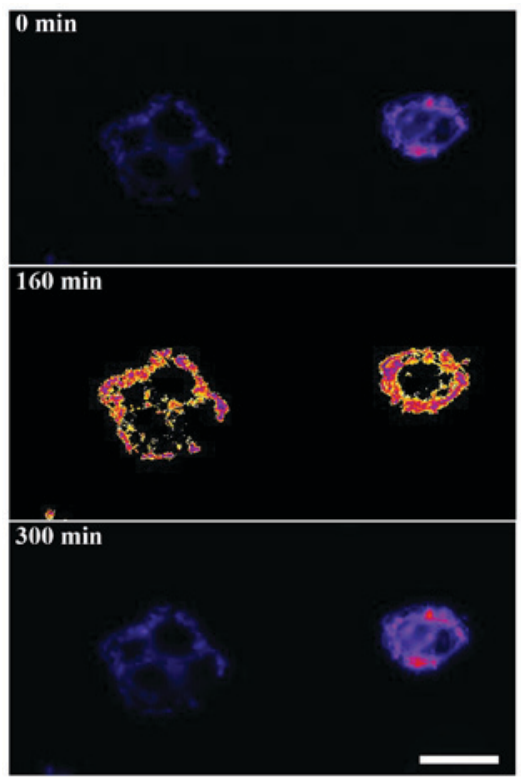

\section{Ratio $405 / 488 \mathrm{~nm}$

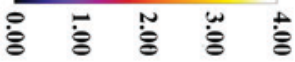

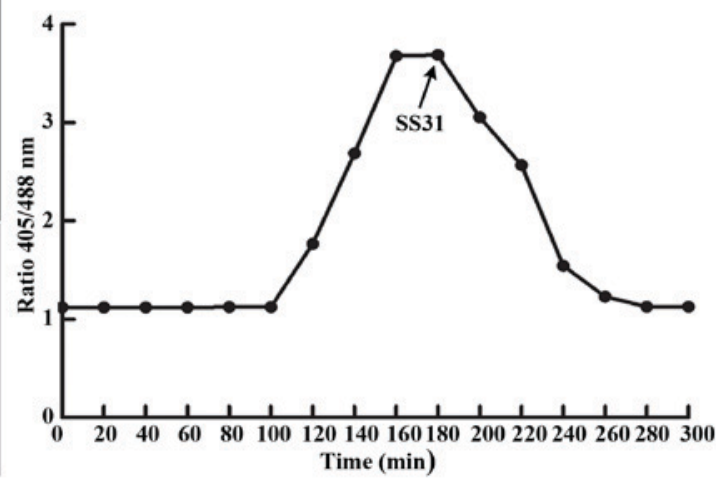

Figure 2. Time-lapse redox response of mitochondria to oridonin and SS31 treatment in HepG2 cells. Cells were treated with $25 \mu \mathrm{M}$ oridonin, followed by $100 \mathrm{nM}$ SS31. The representative images of the mtGrx1-roGFP2 sensor presented were visualized with excitation at 405 and $488 \mathrm{~nm}$ using Nikon A1R confocal laser scanning system (magnification, $\mathrm{x} 400$ ). False-color ratio images of the cells at indicated time points provide visual confirmation of mitochondrial redox changes (left panel). Corresponding ratiometric data were plotted against time (right panel). Scale bar, $10 \mu \mathrm{m}$. Data are representative of 3 experiments.

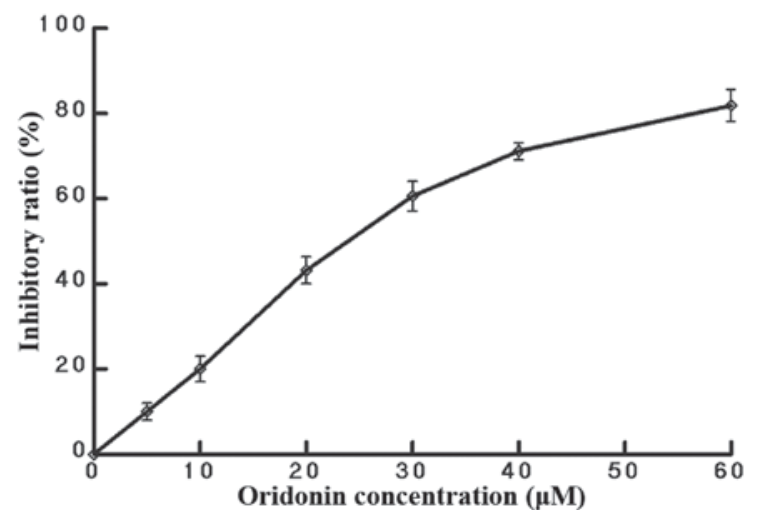

Figure 3. Oridonin inhibits HepG2 cell growth in a concentration-dependent manner. The cells were treated with various doses of oridonin at $0,5,10$, 20,30 or $60 \mu \mathrm{M}$ for $8 \mathrm{~h}$. Cell growth inhibition ratio was measured by a cell counting kit assay. Data are representative of 5 experiments.

As demonstrated in Fig. 4B, nuclear condensation and fragmentation were identified in the HepG2-mtGrx1-roGFP2 cells treated with oridonin, whereas SS31 treatment resulted in the integrity of cell morphology, similar to untreated HepG2 cells. Collectively, these data implicate ROS in the mitochondria as important contributors to oridonin-induced cell apoptosis.

Mitochondrial ROS contribute to oridonin-induced PARP activation in HepG2 cells. Hypothetically, oxidant stress-induced redox changes may trigger the activation of PARP, which serves an indispensable role in commonly described initiation pathways and redundant cell death pathways (41-43). To elucidate the molecular mechanism responsible for oridonin-induced apoptosis mediated by mitochondrial redox changes in HepG2 cells, the expression levels of PARP were determined by western blot analysis. As demonstrated in Fig. 5, cleaved PARP protein expression was markedly increased by oridonin at a concentration of $25 \mu \mathrm{M}$. However, the activation of PARP cleavage was inhibited by SS31 administration. The results additionally confirm that redox signaling in mitochondria serves as a modulator of oridonin-induced apoptosis through the activation of PARP.

\section{Discussion}

Previous studies have indicated that oridonin possesses anti-proliferative and apoptotic activities against a variety of cancer cells $(44,45)$. However, the underlying regulatory mechanisms of mitochondrial ROS in oridonin-induced HepG2 apoptosis remain largely unknown due to limitations of subcellular imaging resolution. Previously, it has been argued that mitochondria are more sensitive to redox perturbation compared with other subcellular compartments, and studies have suggested that mitochondria serve a potential role in sensing and signaling cellular redox challenge in vital biological processes such as cell death and abiotic stress response, based on the mitochondrial-targeted redox-sensitive GFP $(41,46)$. In the present study, the mitochondrial-targeted Grx1-roGFP2 biosensor, which has been demonstrated as a valuable tool in sensing compartmentalized redox status and consequently ROS homeostasis/signaling in human cancer cell lines (47), was applied to monitor temporal changes in the redox status of mitochondria in HepG2 cells in response to either $\mathrm{H}_{2} \mathrm{O}_{2}$ /DTT or oridonin/SS31 (Figs. 1B and 2), indicating that a redox-sensing system in HepG2 cells was successfully established. Based on the regulation of mitochondrial redox changes in oridonin-induced apoptosis (Fig. 4), additional experiments were conducted to elucidate the potential mechanisms of action of mitochondrial redox changes in HepG2 cells, as demonstrated in Fig. 5, which were partially dependent on PARP cleavage. Taken together, the present study provides evidence in support of mitochondrial ROS as a potential 

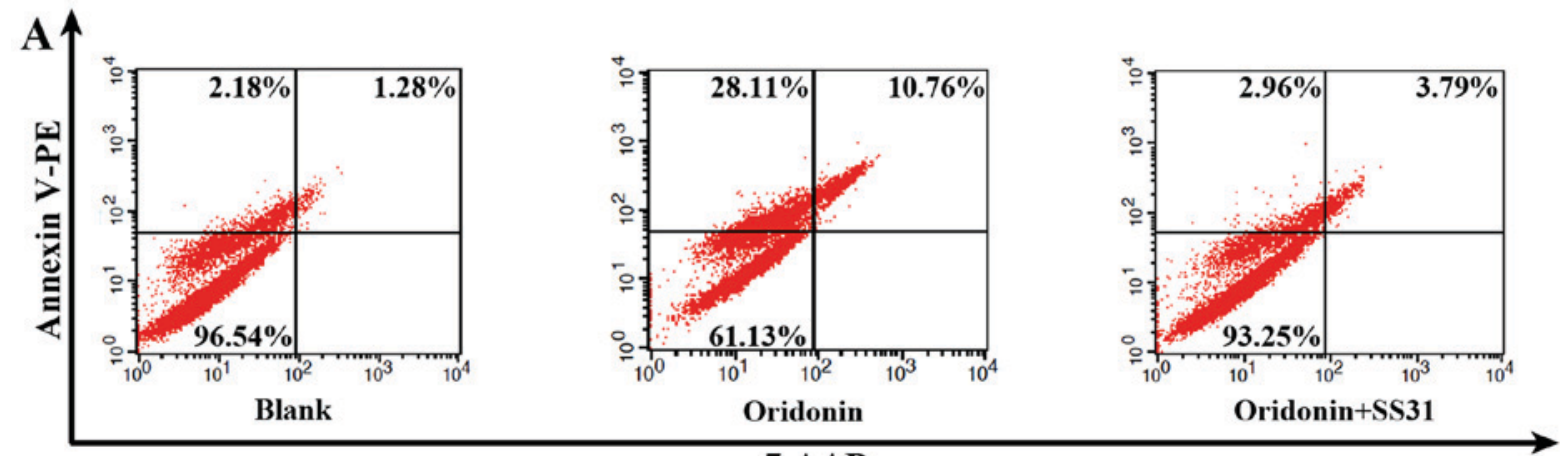

7-AAD

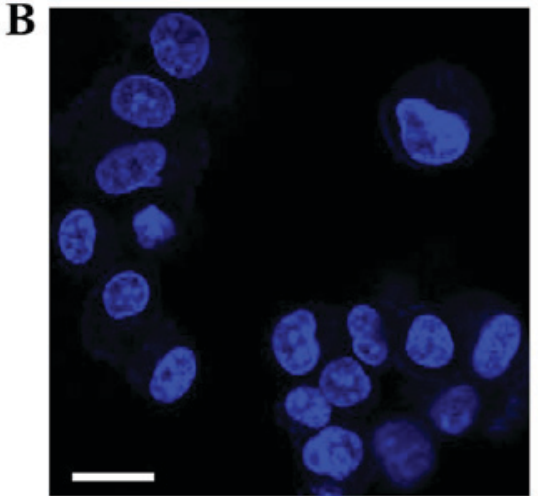

Blank

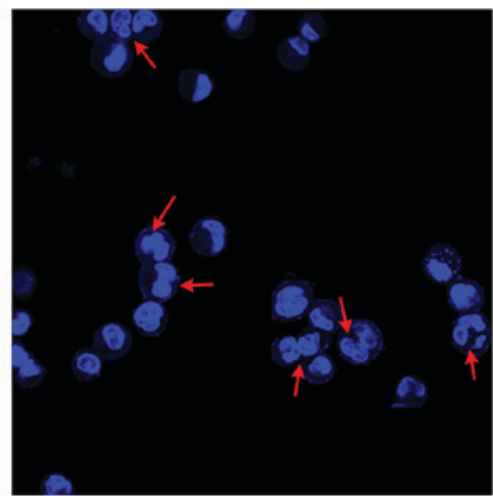

Oridonin

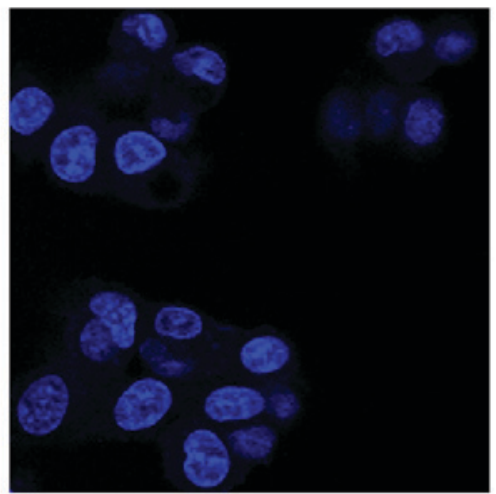

Oridonin+SS31

Figure 4. Oridonin-induced apoptosis is associated with mitochondrial redox changes in HepG2 cells. Cells were incubated with $0 \mu \mathrm{M}$ oridonin (blank), $25 \mu \mathrm{M}$ oridonin (oridonin), or co-incubated with $25 \mu \mathrm{M}$ oridonin and $100 \mathrm{nM} \mathrm{SS} 31$ (oridonin+SS31) for $8 \mathrm{~h}$. (A) The apoptotic ratio of three groups was analyzed by flow cytometric analysis. (B) The cellular morphologic changes of three groups were observed by Olympus FV1000 confocal laser scanning microscopy (magnification, x400). The arrows indicate nuclear condensation and fragmentation. Scale bar, $10 \mu \mathrm{m}$. The results are representative data from 3 experiments. 7-AAD, 7-aminoactinomycin; PE, phycoerythrin.

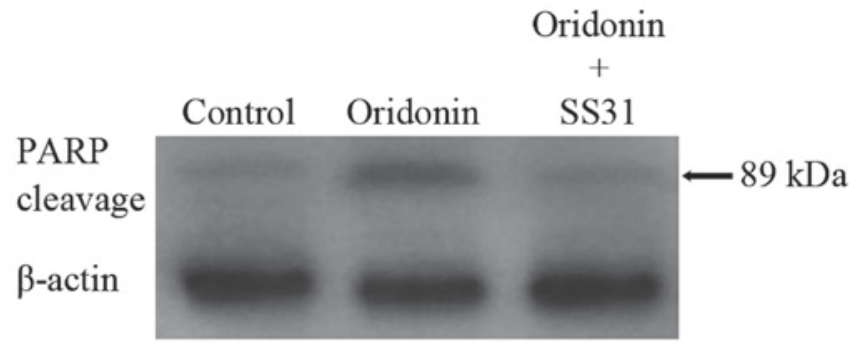

Figure 5. Expression of apoptosis-associated protein PARP is regulated by mitochondrial ROS in HepG2 cells. Cells were treated with $25 \mu \mathrm{M}$ oridonin for $8 \mathrm{~h}$ in the presence or absence of $100 \mathrm{nM} \mathrm{SS31}$. The expression levels of PARP were detected by western blot analysis, and $\beta$-actin was used as a loading control. The results are representative of 3 experiments. PARP, poly adenosine 5'-diphosphate ribose polymerase.

mediator in the apoptotic activities of oridonin in HepG2 cells, which provides insight on the molecular mechanisms by which mitochondrial redox signaling regulates apoptosis in response to oridonin in cancer therapy. Additional investigations are required for the development of mitochondrial-specific oridonin as a novel promising anticancer therapeutic strategy.

Consistent with previous studies $(14,18,20,47)$, the mtGrx1-roGFP2 redox biosensor exhibited excellent specificity and sensitivity, as exemplified by the response to oridonin in HepG2 cells. Future studies should focus on certain key protein in the signaling pathways associated with apoptosis, including tumor protein (p)53 $(35,48)$ using mtGrx1-roGFP2 to determine whether p53 participates in oridonin-induced apoptosis through the generation of mitochondrial ROS. In principle, such redox-sensing systems may be applied to dissect the regulatory mechanisms of redox signaling, or for drug screening and toxicology $(18,21)$. Redox signaling mechanisms associated with HIV and HIV-Mycobacterium tuberculosis co-infection were examined by performing oxidative stress and antioxidant defense pathway-focused human gene expression arrays (RT2 Profiler ${ }^{\mathrm{TM}}$ PCR arrays), using Grx1-roGFP2 (18). Compared with TNF-related apoptosis-inducing ligand (TRAIL) as an acknowledged apoptosis inducer (21), oridonin altered the redox state of the mitochondria to the extent that the mitochondrial sensor demonstrated highly rapid and marked changes in oxidation (Fig. 2), suggesting that the activity of oridonin as a redox-active molecule may be superior to that of TRAIL, and potentially an excellent therapeutic candidate for targeting cancer. However, it should be noted that the activity of the mitochondrial sensor mtGrx1-roGFP2 has not been compared or normalized between HeLa and HepG2 cell lines. Additional studies are required to explore the issue as to which subcellular compartment is more susceptible to redox changes induced by oridonin.

In HepG2 cells, multiple mechanisms, including the inhibition of telomerase, induction of endoplasmic reticulum stress and regulation of p53, MAPK and mitochondrial signaling pathways, have been implicated in the apoptotic activity of oridonin $(25,49,50)$. Whether other mechanisms, such as PARP activation, are associated with the anti-HepG2 activity 
of oridonin remains unknown. To the best of our knowledge, for the first time, the present study investigated the involvement of PARP activated by mitochondrial ROS in contributing to oridonin-induced HepG2 apoptosis (Fig. 5), as previously demonstrated in menadione-triggered cell death pathways (41). This provides further insight into the molecular mechanisms of oridonin function in HepG2 cells.

\section{Acknowledgements}

The authors would like to thank Professor Dick German Cancer Research Center, Heidelberg, Germany for kindly providing the mtGrx1-roGFP2 plasmid. The present study was supported by the Natural Science Fundamental Research Program of Henan Provincial Education Department (grant no. 16A180046) and Zhengzhou Science and Technology Development Plan (grant no. 20150350).

\section{References}

1. Mohan H: Textbook of pathology. 5th edition. Jaypee Brothers Medical Publishers, New Delhi, pp21-60, 2010.

2. Merkle CJ: Cellular adaptation, injury and death. In Pathophysiology: Concepts of altered health states. Porth CM and Matfin $\mathrm{G}$ (eds). 8th edition. Wolters Kluwer/Lippincott Williams and Wilkins, Philadelphia, PA, pp94-111, 2009.

3. Ghobrial IM, Witzig TE and Adjei AA: Targeting apoptosis pathways in cancer therapy. CA Cancer J Clin 55: 178-194, 2005 .

4. Wong RS: Apoptosis in cancer: From pathogenesis to treatment. J Exp Clin Cancer Res 30: 87, 2011.

5. Parri $M$ and Chiarugi P: Redox molecular machines involved in tumor progression. Antioxid Redox Signal 19: $1828-1845,2013$

6. Tochhawng L, Deng S, Pervaiz S and Yap CT: Redox regulation of cancer cell migration and invasion. Mitochondrion 13: 246-253, 2013

7. Zhang L, Wang K, Lei Y, Li Q, Nice EC and Huang C: Redox signaling: Potential arbitrator of autophagy and apoptosis in therapeutic response. Free Radic Biol Med 89: 452-465, 2015.

8. Liao W, Yu Z, Lin Z, Lei Z, Ning Z, Regenstein JM, Yang J and Ren J: Biofunctionalization of Selenium nanoparticle with dictyophora indusiata polysaccharide and its antiproliferative activity through death-receptor and mitochondria-mediated apoptotic pathways. Sci Rep 5: 18629, 2015.

9. Xue M, Ge Y, Yu C, Zheng Z, He X and Zhao J: Apoptosis is induced by docosahexaenoic acid in breast cancer cells via death receptor and mitochondria-mediated pathways. Mol Med Rep 16: 978-982, 2017

10. Zhang YQ, Xiao CX, Lin BY, Shi Y, Liu YP, Liu JJ, Guleng B and Ren JL: Silencing of pokemon enhances caspase-dependent apoptosis via fas- and mitochondria-mediated pathways in hepatocellular carcinoma cells. PLoS One 8: e68981, 2013.

11. Kroemer G: Mitochondrial control of apoptosis: An overview. Biochem Soc Symp 66: 1-15, 1999.

12. Mari M, Morales A, Colell A, Garcia-Ruiz C and Fernandez-Checa JC: Mitochondrial glutathione, a key survival antioxidant. Antioxid Redox Signal 11: 2685-2700, 2009.

13. Morgan B, Sobotta MC and Dick TP: Measuring E(GSH) and $\mathrm{H}_{2} \mathrm{O}_{2}$ with roGFP2-based redox probes. Free Radic Biol Med 51: 1943-1951, 2011

14. Kolossov VL, Hanafin WP, Beaudoin JN, Bica DE, DiLiberto SJ, Kenis PJ and Gaskins HR: Inhibition of glutathione synthesis distinctly alters mitochondrial and cytosolic redox poise. Exp Biol Med (Maywood) 239: 394-403, 2014.

15. Chan CK, Supriady H, Goh BH and Kadir HA: Elephantopus scaber induces apoptosis through ROS-dependent mitochondrial signaling pathway in HCT116 human colorectal carcinoma cells. J Ethnopharmacol 168: 291-304, 2015.

16. Shen S, Zhang Y, Zhang R and Gong X: Sarsasapogenin induces apoptosis via the reactive oxygen species-mediated mitochondrial pathway and ER stress pathway in HeLa cells. Biochem Biophys Res Commun 15: 519-524, 2013.
17. Ezerina D, Morgan B and Dick TP: Imaging dynamic redox processes with genetically encoded probes. J Mol Cell Cardiol 73: 43-49, 2014.

18. Bhaskar A, Munshi M, Khan SZ, Fatima S, Arya R, Jameel S and Singh A: Measuring glutathione redox potential of HIV-1-infected macrophages. J Biol Chem 290: 1020-1038, 2015

19. van Lith M, Tiwari S, Pediani J, Milligan G and Bulleid NJ: Real-time monitoring of redox changes in the mammalian endoplasmic reticulum. J Cell Sci 124: 2349-2356, 2011.

20. Hanson GT, Aggeler R, Oglesbee D, Cannon M, Capaldi RA, Tsien RY and Remington SJ: Investigating mitochondrial redox potential with redox-sensitive green fluorescent protein indicators. J Biol Chem 279: 13044-13053, 2004.

21. Gutscher M, Pauleau AL, Marty L, Brach T, Wabnitz GH, Samstag Y, Meyer AJ and Dick TP: Real-time imaging of the intracellular glutathione redox potential. Nat Methods 5: 553-559, 2008.

22. Kang N, Zhang JH, Qiu F, Tashiro S, Onodera S and Ikejima T: Inhibition of EGFR signaling augments oridonin-induced apoptosis in human laryngeal cancer cells via enhancing oxidative stress coincident with activation of both the intrinsic and extrinsic apoptotic pathways. Cancer Lett 294: 147-158, 2010.

23. Zhang YH, Wu YL, Tashiro S, Onodera S and Ikejima T: Reactive oxygen species contribute to oridonin-induced apoptosis and autophagy in human cervical carcinoma HeLa cells. Acta Pharmacol Sin 32: 1266-1275, 2011.

24. Chen G, Wang K, Yang BY, Tang B, Chen JX and Hua ZC: Synergistic antitumor activity of oridonin and arsenic trioxide on hepatocellular carcinoma cells. Int J Oncol 40: 139-147, 2012.

25. Wang H, Ye Y and Yu ZL: Proteomic and functional analyses demonstrate the involvement of oxidative stress in the anticancer activities of oridonin in HepG2 cells. Oncol Rep 31: 2165-2172, 2014.

26. Yu Y, Fan SM, Ye YC, Tashiro S, Onodera S and Ikejima T: The tyrphostin AG1478 augments oridonin-induced A431 cell apoptosis by blockage of JNK MAPK and enhancement of oxidative stress. Free Radic Res 46: 1393-1405, 2012.

27. Yu Y, Fan SM, Song JK, Tashiro S, Onodera S and Ikejima T: Hydroxyl radical ( $\mathrm{OH}$ ) played a pivotal role in oridonin-induced apoptosis and autophagy in human epidermoid carcinoma A431 cells. Biol Pharm Bull 35: 2148-2159, 2012.

28. Zang L, He H, Xu Q, Yu Y, Zheng N, Liu W, Hayashi T, Tashiro S, Onodera $\mathrm{S}$ and Ikejima T: Reactive oxygen species $\mathrm{H}_{2} \mathrm{O}_{2}$ and $\cdot \mathrm{OH}$, but not $\mathrm{O}_{2} \cdot(-)$ promote oridonin-induced phagocytosis of apoptotic cells by human histocytic lymphoma U937 cells. Int Immunopharmacol 15: 414-423, 2013.

29. Pi J, Cai H, Jin H, Yang F, Jiang J, Wu A, Zhu H, Liu J, Su X, Yang P and Cai J: Qualitative and quantitative analysis of ROS-mediated oridonin-induced oesophageal cancer KYSE-150 cell apoptosis by atomic force microscopy. PLoS One 10: e0140935, 2015.

30. Shang $\mathrm{CH}$, Zhang QQ and Zhou JH: Oridonin inhibits cell proliferation and induces apoptosis in rheumatoid arthritis fibroblast-like Synoviocytes. Inflammation 39: 873-880, 2016.

31. Xu ZZ, Fu WB, Jin Z, Guo P, Wang WF and Li JM: Reactive oxygen species mediate oridonin-induced apoptosis through DNA damage response and activation of JNK pathway in diffuse large B cell lymphoma. Leuk Lymphoma 57: 888-898, 2016.

32. Huang J, Wu L, Tashiro S, Onodera S and Ikejima T: Reactive oxygen species mediate oridonin-induced HepG2 apoptosis through p53, MAPK and mitochondrial signaling pathways. J Pharmacol Sci 107: 370-379, 2008.

33. Bao R, Shu Y, Wu X, Weng H, Ding Q, Cao Y, Li M, Mu J, Wu W, Ding Q, et al: Oridonin induces apoptosis and cell cycle arrest of gallbladder cancer cells via the mitochondrial pathway. BMC Cancer 14: 217, 2014.

34. Li J, Chen X, Xiao W, Ma W, Li T, Huang J, Liu X, Liang X, Tang S and Luo Y: Mitochondria-targeted antioxidant peptide SS31 attenuates high glucose-induced injury on human retinal endothelial cells. Biochem Biophys Res Commun 404: 349-356, 2011.

35. Zhao K, Zhao GM, Wu D, Soong Y, Birk AV, Schiller PW and Szeto HH: Cell-permeable peptide antioxidants targeted to inner mitochondrial membrane inhibit mitochondrial swelling, oxidative cell death and reperfusion injury. J Biol Chem 279: 34682-34690, 2004.

36. Zhao K, Luo G, Giannelli S and Szeto HH: Mitochondria-targeted peptide prevents mitochondrial depolarization and apoptosis induced by tert-butyl hydroperoxide in neuronal cell lines. Biochem Pharmacol 70: 1796-1806, 2005. 
37. López-Terrada D, Cheung SW, Finegold MJ and Knowles BB Hep G2 is a hepatoblastoma-derived cell line. Hum Pathol 40: $1512-1515,2009$

38. Capes-Davis A, Theodosopoulos G, Atkin I, Drexler HG, Kohara A, MacLeod RA, Masters JR, Nakamura Y, Reid YA, Reddel RR and Freshney RI: Check your cultures! A list of cross-contaminated or misidentified cell lines. Int J Cancer 127: $1-8,2010$.

39. Zufferey R, Nagy D, Mandel RJ, Naldini L and Trono D: Multiply attenuated lentiviral vector achieves efficient gene delivery in vivo. Nat Biotechnol 15: 871-875, 1997.

40. Rota C, Chignell CF and Mason RP: Evidence for free radical formation during the oxidation of 2'-7'-dichlorofluorescin to the fluorescent dye 2'-7'-dichlorofluorescein by horseradish peroxidase: Possible implications for oxidative stress measurements. Free Radic Biol Med 27: 873-881, 1999.

41. Loor G, Kondapalli J, Schriewer JM, Chandel NS, Vanden Hoek TL and Schumacker PT: Menadione triggers cell death through ROS-dependent mechanisms involving PARP activation without requiring apoptosis. Free Radic Biol Med 49: 1925-1936, 2010.

42. Ye W, Zhong Z, Zhu S, Zheng S, Xiao J, Song S, Yu H, Wu Q, Lin $\mathrm{Z}$ and $\mathrm{Chen} \mathrm{J}$ : Advanced oxidation protein products induce chondrocyte death through a redox-dependent, poly (ADP-ribose) polymerase-1-mediated pathway. Apoptosis 22: 86-97, 2017.

43. Lu P, Kamboj A, Gibson SB and Anderson CM: Poly(ADP-ribose) polymerase-1 causes mitochondrial damage and neuron death mediated by Bnip3. J Neurosci 34: 15975-15987, 2014.

44. Li CY, Wang EQ, Cheng Y and Bao JK: Oridonin: An active diterpenoid targeting cell cycle arrest, apoptotic and autophagic pathways for cancer therapeutics. Int J Biochem Cell Biol 43 701-704, 2011.
45. Ikezoe T, Chen SS, Tong XJ, Heber D, Taguchi H and Koeffler HP: Oridonin induces growth inhibition and apoptosis of a variety of human cancer cells. Int J Oncol 23: 1187-1193, 2003.

46. Schwarzlander M, Fricker MD and Sweetlove LJ: Monitoring the in vivo redox state of plant mitochondria: effect of respiratory inhibitors, abiotic stress and assessment of recovery from oxidative challenge. Biochim Biophys Acta 1787: 468-475, 2009.

47. Albrecht SC, Sobotta MC, Bausewein D, Aller I, Hell R, Dick TP and Meyer AJ: Redesign of genetically encoded biosensors for monitoring mitochondrial redox status in a broad range of model eukaryotes. J Biomol Screen 19: 379-386, 2014.

48. Johnson TM, Yu ZX, Ferrans VJ, Lowenstein RA and Finkel T: Reactive oxygen species are downstream mediators of p53-dependent apoptosis. Proc Natl Acad Sci USA 93: 11848-11852, 1996.

49. Wang H, Ye Y, Chui JH, Zhu GY, Li YW, Fong DW and Yu ZL: Oridonin induces $\mathrm{G} 2 / \mathrm{M}$ cell cycle arrest and apoptosis through MAPK and p53 signaling pathways in HepG2 cells. Oncol Rep 24: 647-651, 2010.

50. Wang H, Ye Y, Chu JH, Zhu GY, Fong WF and Yu ZL: Proteomic and functional analyses reveal the potential involvement of endoplasmic reticulum stress and alpha-CP1 in the anticancer activities of oridonin in HepG2 cells. Integr Cancer Ther 10: 160-167, 2011.

c) () $\ominus$ This work is licensed under a Creative Commons Attribution-NonCommercial-NoDerivatives 4.0 International (CC BY-NC-ND 4.0) License. 